
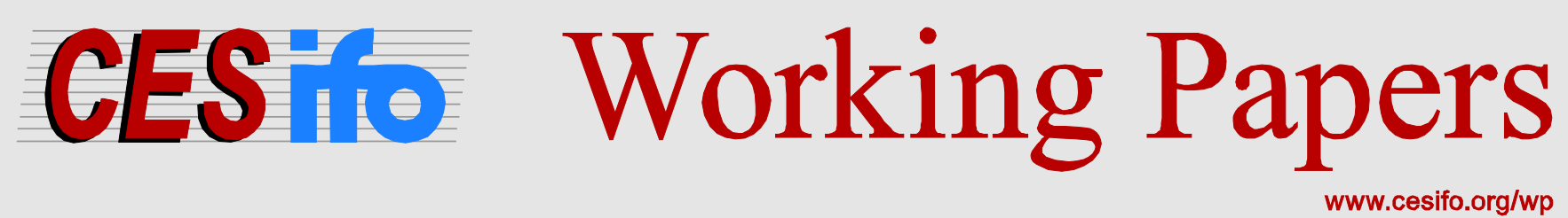

\title{
On the Optimal Target Curbside Parking Occupancy Rate
}

\author{
Richard Arnott
}

CESIFO WORKING PAPER NO. 4416

CATEgory 2: Public CHOICE

SEPTEMBER 2013

An electronic version of the paper may be downloaded

- from the SSRN website:

- from the RePEc website:

- from the CESifo website:

WWW.SSRN.com

Www.RePEc.org

www.CESifo-group.org/wp

\section{CESifo}




\title{
On the Optimal Target Curbside Parking Occupancy Rate
}

\begin{abstract}
For many years, Donald Shoup has been advocating cashing out free and underpriced curbside parking. How should this be implemented in practice, taking into account the stochasticity of curbside parking vacancies? Shoup has proposed setting neighborhood/period of the day-specific meter rates such that a common target (average) curbside parking occupancy rate is achieved. Taking as given how the average occupancy rate affects expected cruising-for-parking time and expected walking time (between parking space and destination), this paper investigates the optimal (surplus-maximizing) target curbside parking occupancy rate. The principal result is that the rate should be higher, the higher is the level of demand.
\end{abstract}

JEL-Code: D600, H200, J600, N700, R400.

Keywords: parking, curbside parking, occupancy rate, vacancy rate, pricing.

\author{
Richard Arnott \\ Department of Economics \\ University of California \\ USA - Riverside, CA 92506 \\ richard.arnott@ucr.edu
}

September 26, 2013

This paper is dedicated to the memory of Herbert Mohring, in recognition of his seminal contributions to urban transport economic theory. Reprint submitted to Elsevier. 


\section{Introduction}

Even on a particular city block at a particular time of day, the number of vacant curbside parking spaces varies substantially from day to day. At a point in time, the block's curbside parking vacancy rate is the realization of complex curbside parking arrival (birth) and departure (death) stochastic processes. There may be a special event on that block that generates a higher than usual arrival rate for a period of time before that event starts, and a concentration of departures when it is over. Or purely by chance, an abnormally high number of people may choose to have lunch at a particular restaurant on that block. To further complicate matters, there is spatial autocorrelation in the vacancy rate on neighboring blocks, as drivers, unable to find curbside parking on their destination block, search/cruise for parking on neighboring blocks.

The stochasticity of vacant curbside parking spaces is practically important. Even when the average vacancy rate on a particular city block at a particular time of day is, say, $10 \%$, there will be days when drivers whose destination is on that block have to spend a substantial amount of time cruising for a curbside parking space. So as to avoid being late for an appointment, a driver may respond to this lack of reliability in curbside parking search time by departing home earlier and/or starting to search for parking well before reaching the destination block.

Vickrey (1954) was the first economist to address the importance of the stochastic nature of the curbside parking vacancy rate. He advocated responsive curbside parking pricing to deal with the phenomenon. The parking meters on a block would be simultaneously monitored, and the curbside parking fee ${ }^{1}$

\footnotetext{
${ }^{1}$ The terms "curbside parking fee" and "meter rate" are used interchangeably.
} 
would be adjusted responsively to achieve an ex post curbside vacancy rate, such that there would almost always be a vacant curbside parking space on each block, which would virtually eliminate the time wasted in cruising for parking and the added congestion it causes. Vickrey's proposal never went beyond the drawing board because it was technologically ahead of its time. Recently Shoup (1999) has been advocating differentiating the curbside meter rate by block and time of day so as to achieve a common target curbside parking occupancy rate (hereafter, target occupancy rate); he has proposed a rate of $85 \%$. Implementing his proposed scheme would require extensive data collection but no high technology. A modified version of his proposed scheme is being implemented on an experimental basis in San Francisco (SFpark.org).

Averaged over time and space, does a target occupancy rate of $85 \%$ maximize social surplus, or should it be higher or lower? Should the target occupancy rate vary depending on the time of day, perhaps being lower in the morning when the expected arrival rate exceeds the expected departure rate, or depending on location, perhaps being higher at locations with shorter average parking durations? This paper takes a first step towards determining the optimal (surplus-maximizing) target occupancy rate analytically. Assume that space is isotropic and that the economy is in stochastic steady state, so that the optimal target occupancy rate is invariant over time and space. Its determination can be viewed as the solution of a problem involving three modules. The first relates to the outcomes of alternative search strategies, taking as given the probability distribution of different patterns of parking occupancy over space. The second derives the probability distribution of different patterns of occupancy over space from the stochastic processes determining trip generation and termination, under alternative search strategies. The third derives the surplusmaximizing target vacancy rate under alternative search strategies. Solution 
of this problem would be formidably difficult. To generate a problem that is manageable, I construct a model, building on that in Arnott and Rowse (1999), which assumes that parking spaces are uniformly distributed around the circumference of a circle, trip originations are generated by a time-invariant Poisson process at a rate that is uniform around the circle, the distance between trip origins and destinations is constant, travel occurs in only one direction, and the visit length at the destination is constant. Under these assumptions, a driver's search strategy is simple: after initiating search, take the first vacant parking space encountered. A driver then has only two decisions to make, how long before her appointment time to initiate her trip, and how far before her destination to initiate search. The probabilities of encountering the first vacant space at the first parking space, the second parking space, and so on, after parking search is initiated, as a function of the average occupancy rate (averaged over time and space), can then be solved for, at least computationally.

The parking planner controls the curbside occupancy rate only indirectly via the curbside meter rate. Adding a demand function relating the Poisson rate at which trips are initiated to the expected full price of a trip permits determination of equilibrium and social surplus, as functions of the curbside meter rate, and thence of the optimal curbside meter rate and the optimal target occupancy rate.

Because the underlying stochastic processes generating the actual patterns of curbside parking occupancy vary over both space and time and are much more complex than the simple stochastic process assumed in the model, and because actual two-dimensional parking search strategies are much more complex than the simple one-dimensional search strategy implied by our model, considerable work - data collection and analysis, and theoretical development - will need to be done before optimal target occupancy rates can be determined in policy practice. 
I hope nonetheless that this paper adds value in providing some conceptual foundation for their determination.

Section 2 provides a brief review of relevant literature. Section 3 presents the model. Section 4 derives analytically the optimal target curbside occupancy rate, taking as given the probability distribution of the number of occupied curbside parking spaces searched prior to finding a vacant space, conditional on the average occupancy rate. Section 5 discusses directions for future research, and section 6 concludes.

\section{Literature review}

Several papers have investigated models of rush-hour traffic dynamics in which individuals have a common desired arrival time at a common destination, are perfectly informed about the availability of curbside parking spaces on a radial artery, and can choose between vacant parking spaces. Arriving earlier provides a larger choice set of vacant parking spaces but increases schedule delay. Arnott et al. (1991) considered such a model of morning rush-hour travel to a common downtown location, with bottleneck congestion upstream of downtown parking spaces. By varying the curbside parking fee across time and location, the planner can control the order in which parking spaces are occupied, which affects the time pattern of congestion at the bottleneck. Zhang et al. (2008), Zhang et al. (2011), and Qian et al. (2012) provide various extensions of Arnott et al. (1991) to examine alternative downtown parking policies. Anderson and de Palma (2004) explore a model similar to Arnott et al. (1991) without a bottleneck but with congestible parking on side streets, one section of which considers cruising for parking.

Arnott and Inci (2006, 2010), Arnott and Rowse (2009, forthcoming), and Arnott et al. (2013) present a series of related models that investigate the interaction between cruising for underpriced curbside parking and traffic congestion 
in an isotropic downtown area in stationary state. Curbside parking spaces reduce the road space available for travel, and cars cruising for parking contribute to traffic congestion. Arnott and Inci (2006) explores a model with only curbside parking, and Arnott and Inci (2010) examines the stability of the model's equilibria. Arnott and Rowse (2009) considers a model with both curbside and garage parking, and considers spatial competition between parking garages, and Arnott and Rowse (forthcoming) extends that model to consider curbside parking time limits and heterogeneity among parkers. Arnott et al. (2013) presents an integrative diagrammatic analysis focusing on first- and second-best optimal curbside parking capacity. All the above Arnott, Inci, and/or Rowse papers assume that expected cruising for parking search time is given by $C L / P$ where $C$ is the density per unit area of cars cruising for parking, $L$ is parking duration, and $P$ is the density of curbside parking spaces, and that walking time between the parking space and destination is zero. This specification assumes that, at all locations, parking spaces become available to a driver who is cruising for parking according to a spatially uniform and time-invariant Poisson process with rate $P /(C L)$. Under this assumption, the optimal search strategy is to drive to the destination block and wait until a space becomes available.

Arnott and Rowse (1999) is the first economics paper to investigate curbside parking search at a microscopic level. Individuals and curbside parking spaces are uniformly distributed around the circumference of a circle. An individual waits at home for offers, each of which provides a fixed award, which can be collected by going to a specified, stochastically determined location and remaining there for a fixed period of time. Upon receipt of an offer, she must immediately decide whether to take it up. Conditional on taking it up, she departs immediately, decides how far from her destination to start cruising for parking, and then takes the first vacant parking space, walking from there to her destination. 
Having collected the award, she returns to her parking space, drives home, and awaits the next offer. The paper solved for equilibrium curbside parking occupancy rates, demonstrating possible multiplicity of equilibria. A weakness of the paper, which the authors recognized, is that, to achieve tractability it assumes that the probability that a particular curbside parking space is vacant equals the average curbside parking vacancy rate; that is, the authors assumed away the spatial autocorrelation of occupied curbside parking spaces.

Figure 1: Average cruising time as a function of the parking occupancy rate. PARKANALYST vs PARKAGENT.

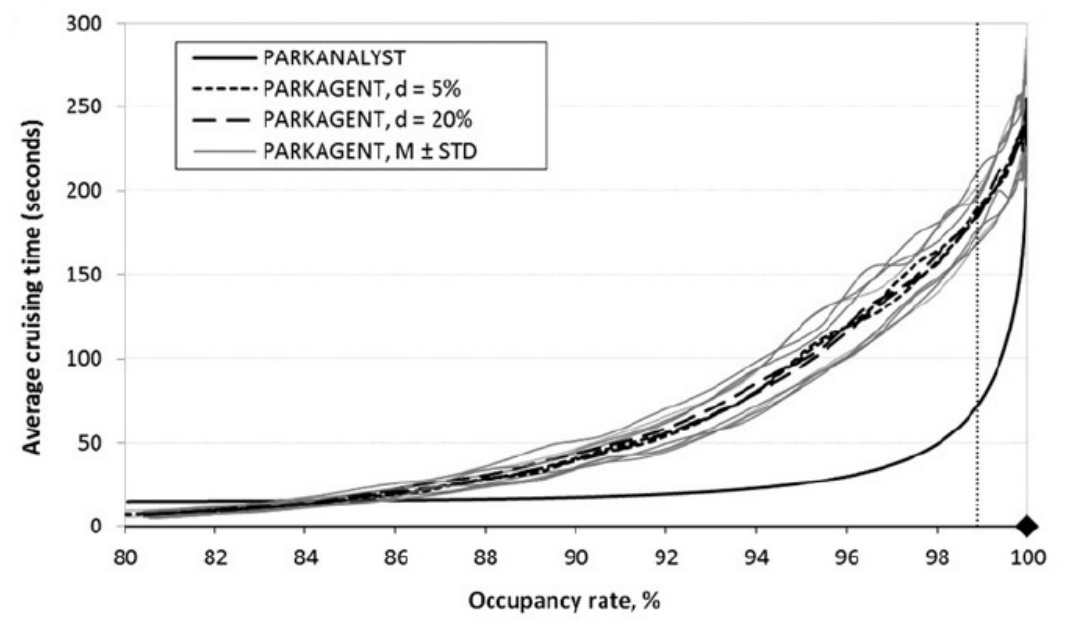

This figure is reproduced from Figure 4 of Levy et al. (2012). In the current context, the difference between the various PARKAGENT graphs is unimportant.

The importance of the spatial autocorrelation of occupied curbside parking spaces is the focus of Levy et al. (2012). They compare the results of an analytical model of parking search similar to Arnott and Rowse (1999), PARKAGENT, in which every driver is confronted with averaged conditions, with the results of a traffic microsimulation model, PARKANALYST, which treats the spatial autocorrelation of occupied curbside parking spaces. Their simulation results demonstrate the quantitative importance of taking into account this 
spatial autocorrelation. Figure 1 below reproduces Figure 4 of their paper. It plots average cruising for parking time against the average occupancy rate. In PARKAGENT, parking search time becomes significant only when the average occupancy rate is close to $100 \%$ (for example, with a occupancy rate of $99 \%$, a driver cruising for parking would expect to drive by 99 occupied parking spaces before locating a vacant space, while with a parking occupancy rate of $90 \%$, a driver cruising for parking would expect to drive by only 9 occupied parking spaces). In PARKANALYST, in contrast, parking search time starts to be nonnegligible with an average occupancy rate of about $85 \%$, and at around $93 \%$ is approximately the same as that in PARKAGENT with a 99\% rate.

\section{The Model}

Consider an isotropic spatial economy organized on the circumference of a circle (an "atoll economy") of infinite radius. Trips are originated around the circle at a uniform Poisson rate that is determined endogenously. Each trip entails travel in one direction ${ }^{2}$ around the circle to a destination a distance $\delta$ from where the trip originated, a visit at the destination of duration $L$ at an appointed time, followed by a return journey to the trip origin. All trips are by car, and a driver must park her car curbside in the vicinity of her destination, and walk from her parking location to her destination, and later back again before driving back to the trip origin. Curbside parking spaces are uniformly distributed around the circle with density $P$ per unit length. From experience, the driver knows the probability distribution of the number of occupied parking spaces she will encounter after initiating cruising for parking before finding a vacant parking space. But she has no information on the realized configuration of occupied parking spaces at the time she commences her outbound journey and

\footnotetext{
${ }^{2}$ Allowing a car to turn around after it has passed the destination complicates the algebra without adding insight.
} 
receives no information during her journey. Furthermore, she does not exploit information from the pattern of occupied parking spaces that she encounters on her journey to update the probability distribution. Under these assumptions, there is a single rational search strategy. Start searching for parking a distance $x$ from the destination, where $x$ is chosen by the driver to minimize trip cost, and take the first vacant parking space. There is a curbside parking fee of $f$ per unit time parked. To simplify, traffic congestion is ignored. In-transit travel speed is $v$, cruising-for-parking speed is $s$, and walking speed is $w$ with $v>s>w$.

Each trip involves an appointment at a specified time. If the driver arrives at the appointment early or late, she encounters a schedule delay cost. The $\alpha-\beta-\gamma$ treatment of the value of time is employed; each unit of travel time costs her $\alpha$ (whether in transit, cruising for parking, walking, or visiting at the destination), each unit of time early costs her $\beta$, and each unit of time late costs her $\gamma$. Consistent with intuition and the empirical literature, it is assumed that $\gamma>\beta$. She decides how long before the specified appointment time, $e$, to initiate the trip, as well as $x$, so as to minimize the expected full price of a trip, $F$, which includes the cost of time on the trip, schedule delay cost, and the curbside parking payment. The expected trip duration equals the expected time spent on the trip to the destination, the time spent at the destination, and the expected time spent on the return trip. The expected time spent on the trip to the destination equals the time driving before initiating cruising for parking, plus the expected time cruising for parking, plus the expected time spent walking from the curbside parking space to the destination. The expected time spent on the return trip equals the expected time spent walking from the destination to the curbside parking space plus the expected return driving time. Expected parking duration equals the time spent at the destination plus the expected time walking from the curbside parking space to the destination and 
back again.

We now derive expressions for the components of the individual's full trip price. $z$ is cruising-for-parking distance, a continuous random variable ${ }^{3}$ whose probability distribution depends on the parking occupancy rate, $Q: g(z ; Q)$. It is assumed that the probability distribution is monotonically decreasing $\left(g_{z}(z ; Q)<\right.$ 0 ) and that an increase in the occupancy rate decreases the probability that the first vacant parking spot is found within a given distance of cruising for parking $\left(G_{Q}(z ; Q)<0\right)$.

Given the informational assumptions, expected cruising-for-parking distance is $E z(Q)=\int_{0}^{\infty} z g(z ; Q) d z$. Since cruising-for-parking speed is $s$, expected cruising-for-parking time is

$$
E S(Q)=\frac{E z(Q)}{s}
$$

A driver initiates cruising for parking a distance $x$ prior to reaching her destination. Thus, she may park either before or after reaching her destination. Walking distance between the parking location and the destination (and, on the return journey, between the destination and the parking location) is therefore $|z-x|$. Since walking speed is $w$, the corresponding expected walking time for the entire journey, $E W$, is

$$
E W(x ; Q)=2 \int_{0}^{x} \frac{x-z}{w} g(z ; Q) d z+2 \int_{x}^{\infty} \frac{z-x}{w} g(z ; Q) d z
$$

Since a driver's destination is a fixed distance $\delta$ from the location at which the trip is originated, and since in-transit travel speed is $v$, the in-transit travel time to the destination prior to initiating cruising for parking is $(\delta-x) / v$. The return in-transit travel distance equals the distance of the parking space from

\footnotetext{
${ }^{3}$ In fact, $z$ is a discrete random variable, corresponding to the discreteness of parking locations, but to simplify the algebra we shall treat it as continuous.
} 
the trip origin, which equals $\delta-x+z$. Thus, the expected return in-transit travel time ${ }^{4}$ is $(\delta-x+E z(Q)) / v$, and the total expected in-transit travel time, $E I$, is

$$
E I(x ; Q)=\frac{2(\delta-x)+E z(Q)}{v}
$$

The expected duration of the trip is the sum of expected cruising-for-parking time, expected walking time, expected in-transit travel time, and visit time. The expected time cost of a trip, ETTC, equals the expected duration of a trip times the value of time. Thus,

$$
\operatorname{ETTC}(x ; Q)=\alpha[E S(Q)+E W(x ; Q)+E I(x ; Q)+L]
$$

To calculate expected schedule delay cost, time is measured relative to the appointment time; viz. $t=0$ is appointment time. A driver departs at $t=-e$ and arrives at the destination after driving to a distance $x$ before the destination, cruising for parking, and then walking from the parking space to the destination. Thus, the arrival time, $a$, as a function of $x, e$, and $z$ is given by

$$
a(x, e, z)=-e+\frac{\delta-x}{v}+\frac{z}{s}+\frac{|z-x|}{w}
$$

The schedule delay cost is - $\beta a$ with early arrival and $\gamma a$ with late arrival. A driver chooses $e$ such that she arrives early with some probability, and there is always some probability of late arrival.

There are two cases to consider. In the first, the driver who finds a parking space as soon as she starts cruising for parking arrives early. Since cruising-forparking speed exceeds walking speed, in this case the driver who finds a parking

\footnotetext{
${ }^{4}$ To simplify the algebra, I ignore the possibility that the driver may choose to start cruising for parking as soon as she leaves home. With a very high occupancy rate, this may be optimal. Thus, I am implicitly assuming that this does not occur with the optimal target curbside occupancy rate.
} 
space right at the destination arrives even earlier. In the second case, the driver who finds a parking space as soon as she starts cruising for parking arrives late, but the driver who finds a parking space right at the destination arrives early ${ }^{5}$. I treat only the first case in the paper, since I judge it to be the more realistic ${ }^{6}$. The first case applies under the following assumption:

A-1: $a(x, e, 0)=-e+\frac{\delta-x}{v}+\frac{x}{w}<0$.

The primitive condition under which this case applies is derived in Appendix B. Let $M(x, e)$ denote the $z$ corresponding to on-time arrival, as a function of $\mathrm{x}$ and e, i.e. $a(x, e, M(x, e))=0$. Under $\mathrm{A}-1$, as $z$ increases, the driver arrives increasingly early up to $z=x$, after which she arrives decreasingly early, until she arrives on time at $z=M(x, e)$, after which she arrives increasingly late. When $z<x$, her walking time is $(x-z) / w$ and she arrives early; when $x<z<M(x, e)$, her walking time is $(z-x) / w$ and she arrives early; when $z>M(x, e)$, her walking time is $(z-x) / w$ and she arrives late. Thus, $G(x ; Q)$ is the probability that a driver finds a curbside parking space before reaching her destination, and $G(M(x, e) ; Q)$ is the probability that she arrives at her destination early.

Using (5), her expected schedule delay costs are

\footnotetext{
${ }^{5}$ It might appear that there is a third case in which both a driver who finds a parking space as soon as she starts cruising for parking and a driver who finds a parking space right at the destination arrive late. Then all drivers would arrive late. But with the cost of time early being lower than the cost of time late, which has been assumed, this cannot be optimal since a driver's expected trip cost would be reduced by departing earlier.

${ }^{6}$ I have no data to support this judgment. It seems counter-intuitive that a driver who finds a parking space as soon as she starts cruising for parking would arrive late. That it seems so suggests that this case is uncommon.
} 


$$
\begin{aligned}
E S D C(x, e ; Q)= & \beta \int_{0}^{x}\left[e-\frac{\delta}{v}+z\left(\frac{1}{w}-\frac{1}{s}\right)-x\left(\frac{1}{w}-\frac{1}{v}\right)\right] g(z ; Q) d z \\
& +\beta \int_{x}^{M(x, e)}\left[e-\frac{\delta}{v}-z\left(\frac{1}{w}+\frac{1}{s}\right)+x\left(\frac{1}{w}+\frac{1}{v}\right)\right] g(z ; Q) d z \\
& +\gamma \int_{M(x, e)}^{\infty}\left[-e+\frac{\delta}{v}+z\left(\frac{1}{w}+\frac{1}{s}\right)-x\left(\frac{1}{w}+\frac{1}{v}\right)\right] g(z ; Q) d z
\end{aligned}
$$

where

$$
M(x, e)=\left[e-\frac{\delta}{v}+x\left(\frac{1}{v}+\frac{1}{w}\right)\right]\left[\frac{1}{s}+\frac{1}{w}\right]^{-1}
$$

Her expected curbside parking payment is $f(L+E W(x ; Q))$.

\section{Analysis of the Model}

This section looks first at the driver's optimization problem, then at equilibrium, and then at the social optimum and its decentralization.

\subsection{A Driver's Optimization Problem}

A driver has two decision variables, $e$ the length of time prior to her appointment that she initiates her inbound trip, and $x$ the distance from her destination at which she initiates cruising for parking. She chooses $e$ and $x$, taking as given the average occupancy rate, $Q$, and the probability distribution $g(z ; Q)$ associated with it, so as to minimize the expected full price of a trip. The expected full price of a trip equals the expected opportunity cost of the trip time, ETTC, plus the expected schedule delay cost, ESDC, plus the expected curbside parking payment, which equals the per-unit-time curbside parking fee times the expected parking duration, which equals the visit duration plus expected walking time. Thus, the driver's optimization problem is

$$
\min _{x, e} \hat{F}(x, e ; Q)=\operatorname{ETTC}(x ; Q)+E S D C(x, e ; Q)+f(L+E W(x ; Q))
$$


Note that $e$ enters only ESDC.

Using (6), the first-order condition with respect to $e$ is

$$
\beta G(M(x, e) ; Q)-\gamma(1-G(M(x, e) ; Q))=0 \text { or } G(M(x, e) ; Q)=\frac{\gamma}{\beta+\gamma}
$$

$G(M(x, e) ; Q)$ is the probability of a driver arriving early. Increasing $e$ by one unit results in arrival at the destination a unit time earlier, conditional on being early, with an expected increase in schedule delay cost of $\beta G(M(x, e) ; Q)$, and arrival at the destination a unit time less late, conditional on being late, with an expected decrease in schedule delay cost of $\gamma(1-G(M(x, e) ; Q))$. The driver chooses $e$ to equalize these two magnitudes. Thus, if the unit time late cost is four times the unit time early cost, which roughly accords with the estimated magnitudes, the driver chooses e so that she will arrive early $80 \%$ of the time.

Throughout the paper we use subscripts to denote partial derivatives. The first-order condition with respect to $x$ is

$$
E T T C_{x}+E S D C_{x}+f E W_{x}=0
$$

where, from (1) to (4),

$$
\begin{gathered}
E T T C_{x}=\alpha\left[E W_{x}+E I_{x}\right]=\alpha\left[\frac{4 G(x ; Q)-2}{w}-\frac{2}{v}\right] \\
E W_{x}=\frac{4 G(x ; Q)-2)}{w}
\end{gathered}
$$

and from (6) (the derivation is given in Appendix A), 


$$
\begin{aligned}
E S D C_{x}= & -\beta\left(\frac{1}{w}-\frac{1}{v}\right) G(x ; Q) \\
& +\beta\left(\frac{1}{w}+\frac{1}{v}\right)(G(M(x, e) ; Q)-G(x ; Q)) \\
& -\gamma\left(\frac{1}{w}+\frac{1}{v}\right)(1-G(M(x ; e) ; Q))
\end{aligned}
$$

Combining (10) - (12) gives

$$
\begin{aligned}
& (\alpha+f) \frac{4 G(x ; Q)-2}{w} \\
& -\frac{2 \alpha}{v}-\beta\left(\frac{1}{w}-\frac{1}{v}\right) G(x ; Q) \\
& +\beta\left(\frac{1}{w}+\frac{1}{v}\right)(G(M(x, e) ; Q)-G(x ; Q)) \\
& -\gamma\left(\frac{1}{w}+\frac{1}{v}\right)(1-G(M(x ; e) ; Q))=0
\end{aligned}
$$

$G(x ; Q)$ is the probability that a driver finds parking before reaching her destination (and therefore arrives early), $G(M(x, e) ; Q)-G(x ; Q)$ is the probability that she parks beyond her destination and is early, and $1-G(M(x, e) ; Q)$ is the probability that she arrives late (and therefore parks beyond her destination). A unit increase in $x$ results in the driver: $a$ ) Walking two units distance further, conditional on finding parking before reaching her destination, and two units of distance less far, conditional on not finding parking until after passing her destination, and therefore in a total of $4 G(x ; Q)-2$ units further, resulting in an increase in expected walking and parking cost of $(\alpha+f)(4 G(x ; Q)-2) ; b)$ Driving in transit two units distance less far, at a saving in in-transit travel time cost of $2 \alpha / v ; c)$ Conditional on parking prior to reaching her destination (and therefore arriving early), experiencing a decrease in time early cost of $\beta(1 / w-1 / v)$; conditional on parking beyond her destination and arriving early, incurring an increase in time early cost of $\beta(1 / w+1 / v)$; and conditional on arriving late 
(and therefore parking beyond her destination), experiencing a decrease in time late cost of $\gamma(1 / w+1 / v)$. Combining (9) and (13) yields

$$
G(x ; Q)=\frac{(\alpha+f)+\frac{\alpha w}{v}}{2(\alpha+f)-\beta}
$$

The solution to the driver's optimization problem is given by (7), (9), and (14), which provides three equations in three unknowns, $x, e$, and $M$. We write the solutions in compact form as $x=x(Q, f)$ and $e=e(Q, f)$. Letting $F$ denote the minimized full trip price, we have that

$$
\begin{aligned}
F= & \operatorname{ETTC}(x(Q, f) ; Q) \\
& +\operatorname{ESDC}(x(Q, f), e(Q, f) ; Q) \\
& +f(E W(x(Q, f) ; Q))
\end{aligned}
$$

which is obtained by substituting $x=x(Q, f)$ and $e=e(Q, f)$ into (8). The equation gives the technological relationship between the full trip price and the occupancy rate. We refer to it as the technology function.

The comparative static properties of the drivers's optimization problem, with the occupancy rate held constant, are recorded in table 1.

Table 1: Comparative static properties of the driver's optimization problem

\begin{tabular}{ccccccccccc}
\hline & $f$ & $Q$ & $\alpha$ & $\beta$ & $\gamma$ & $v$ & $s$ & $w$ & $\delta$ & $L$ \\
\hline \hline$x$ & - & + & $?^{a}$ & + & 0 & - & 0 & + & 0 & 0 \\
\hline$e$ & + & $?$ & $?^{a}$ & - & + & + & - & - & 0 & 0 \\
\hline$M(x, e)$ & 0 & + & 0 & - & + & 0 & 0 & 0 & 0 & 0
\end{tabular}

${ }^{a} x_{\alpha}$ has the same sign as $f w / v-\beta(1+w / v)$, and $e_{\alpha}$ is opposite in sign to $x_{\alpha}$.

The derivations are provided in Appendix C. Here we discuss the comparative statics properties of the driver's optimization problem with respect to two variables of particular interest, $f$ and $Q$. 
How $x$ depends on exogenous parameters can be determined from (13). $x_{f}<$ 0 . The intuition is as follows. A rise in the curbside meter rate causes a driver to place relatively more weight on reducing expected parking time and relatively less on reducing expected travel time and expected schedule delay. With an infinite curbside meter rate, $x$ is chosen to minimize expected walking time, which is achieved with $x$ such that $G(x ; Q)=1 / 2$. But with a finite meter rate, $x$ is chosen so that $G(x ; Q)>1 / 2$, which implies initiating cruising for parking farther from the destination than with $G(x ; Q)=1 / 2$, since doing so reduces both expected in-transit travel time and expected schedule delay cost. It can also be shown, as intuition suggests, that $x_{Q}>0$.

From (9), holding $Q$ fixed, $M(x, e)$, the distance a driver cruises for parking for on-time arrival, is independent of $f$. Thus, $M_{x} x_{f}+M_{e} e_{f}=0$. Since from (7) $M_{x}>0$ and $M_{e}>0$, and since $x_{f}<0, e_{f}>0 . e_{Q}$ is of ambiguous sign, depending on how an increase in $Q$ affects $G_{Q}(z ; Q) / g(z ; Q)$ when evaluated at $z=x$ compared to at $z=M(x, e)$.

\subsection{Stochastic Steady-State Equilibrium}

Equilibrium is determined as the solution of two equations in two unknowns. The first, the technology function, relates the full trip price to the occupancy rate and is given by (15), but with $Q$ endogenous. The second is the stochastic steady-state condition that, in expectation, the demand for curbside parking time per unit distance-time, which equals the rate at which trips are initiated per unit distance-time times the average curbside parking duration, equals the expected number of occupied parking spaces per unit length. The rate at which trips are initiated per unit time-distance, $D$, depends on the full trip price: $D=D(F)$. Expected curbside parking duration equals visit duration plus expected time spent walking from the curbside parking space to the destination and back again, $L+E W(x(Q, f), Q)$, where $E W(x, Q)$ is given by (2) and 
$x(Q, f)$ was obtained in the previous subsection. Thus:

$$
D(F)(L+E W(x(Q, f), Q))=Q P .
$$

Since $F=D^{-1}(Q P /(1+E W(x(Q, f), Q)))$, this condition relates the willingness to pay for a trip to the occupancy rate.

It is tempting to interpret (15) as a supply curve and (16) as a demand curve. But the relevant measure of quantity is throughput, not occupancy. Throughput, $r$, is the steady-state rate at which cars enter and exit the system per unit length, and is related to occupancy according to

$$
r=r(Q, f)=\frac{Q P}{(L+E W(x(Q, f), Q))}
$$

so that (16) may be written as $D(F)=r$, which is a conventional demand relation.

Figure 2: Graphical procedure for calculating the stochastic steady-state equilibria

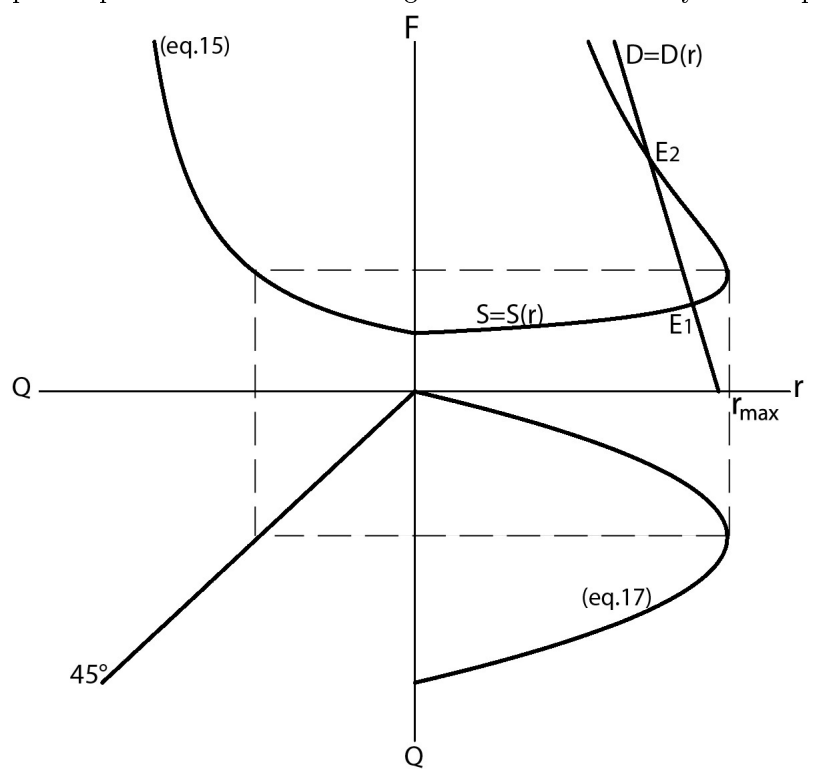

Note: The diagram is qualitative. It is not drawn by graphing functions of the model for specific parameter values.

While I shall work with (15) and (16) in the algebraic analysis, in the di- 
agrammatic analysis I shall focus on $r-F$ space. Figure 2 presents a fourquadrant diagram. Quadrant II displays (15), which relates $F$ to $Q$. Quadrant IV displays (17), which relates $r$ to $Q$. Quadrant III is the 45-degree line. Quadrant $I$ is the panel of particular interest, since it permits a supply-demand interpretation of (15) and (16). The demand curve is an ordinary demand curve, and slopes downward. The supply curve has upward-sloping and backward-bending portions, and is akin to the supply curve of traffic congestion (Walters (1961)), for which the upward-sloping portion corresponds to congested traffic flow and the backward-bending portion to hypercongested traffic flow. The situation here is almost ${ }^{7}$ completely analogous, except that the congestion occurs in parking rather than in traffic flow. Thus, I shall refer to congested and hypercongested parking. Parking is congested (hypercongested) if the elasticity of expected parking duration with respect to the occupancy rate is less than (greater than) one. To understand why the supply curve has the shape it does, consider the extreme situation where the parking occupancy rate is close to $100 \%$. Expected walking distance from the curbside parking space to the destination is very long, resulting in a very long expected parking duration, and hence a very low throughput.

As drawn, there are three equilibria, $E_{1}, E_{2}$, and $E_{3}$. Following the analysis in Arnott and Inci (2010), $E_{1}$ is congested and stable ${ }^{8}, E_{2}$ is hypercongested and unstable, and $E_{3}$, which cannot be displayed in Figure 2 and which corresponds to gridlock but here gridlocked parking rather than gridlocked traffic, is hypercongested and stable. Gridlocked parking corresponds to a situation where all parking spaces are occupied; as a result, cruising-for-parking time and

\footnotetext{
${ }^{7}$ The analogy is not complete since here, unlike in Walters (1961), analysis of traffic congestion where the individual driver chooses only trip frequency, the individual driver chooses not only trip frequency but also $x$ and $e$.

${ }^{8} \mathrm{It}$ is possible for $E_{1}$ to be hypercongested. I ignore this possibility.
} 
walking times are infinite, which results in a zero inflow rate. Gridlock is an equilibrium since both the quantity of throughput demanded and the quantity of throughput supplied is zero.

The comparative static derivatives are complicated since there are three potential channels through which a change in an exogenous parameter affects an endogenous variable, through $x, e$, and $Q$. Appendix $\mathrm{D}$ derives the comparative static derivatives with respect to $f$ and $\eta$, where $\eta$ is a demand shifter, which I term "demand intensity", with higher $\eta$ corresponding to a higher demand curve. The comparative static properties of the stable, congested equilibrium, $E_{1}$, are given in Table 2.

Table 2: Comparative static properties of the stable, congested equilibrium, $E_{1}$

\begin{tabular}{ccc}
\hline & $f$ & $\eta$ \\
\hline \hline$Q$ & - & + \\
\hline$r$ & $?^{a}$ & + \\
\hline$F$ & $?^{a}$ & + \\
\hline
\end{tabular}

${ }^{a}$ The signs of $r_{f}$ and $F_{f}$ are opposite.

The effects of an increase in demand on the stable, congested equilibrium can be seen from Figure 2. The demand curve shifts out, and the supply curve does not change position. Thus, the increase in demand unambiguously increases both throughput and the full price of a trip. Furthermore, since throughput and the occupancy rate are positively related when parking is congested, the increase in demand unambiguously increases the occupancy rate.

The effects of an increase in the curbside parking fee on the stable, congested equilibrium are more complicated. The parking fee increase shifts the supply curve but has no effect on the demand curve. The immediate effect of a unit increase in the parking fee is to shift the supply curve up by the expected parking duration, $L+E W(x(Q, f), Q)$. But the parking fee also affects $x$ directly and through $Q$, and $Q$ directly, all of which change parking duration. It is proven 
in Appendix D that $d Q / d f<0$; an increase in the parking fee unambiguously decreases the occupancy rate of the stable, congested equilibrium. However, $d r / d f$ and $d F / d f$ are ambiguous in sign, though of opposite sign to one another. From (17), $d r / d f=r_{f}+r_{Q} d Q / d f$. Since the equilibrium is congested, $r_{Q}>0$, and since we have obtained that $d Q / d f<0, r_{Q} d Q / d f<0$. But $r_{f}=-[r /(L+$ $E W(x(Q, f), Q))] E W_{x} x_{f}$, which since $E W_{x}>0$ and $x_{f}<0$, is positive. Thus, a rise in the curbside parking fee can lead to an increase in throughput and hence in consumer surplus. A rise in the curbside parking fee can even lead to a simultaneous increase in consumer surplus and parking fee revenue - a double dividend result.

\subsection{Social Optimum}

The social optimum occurs where the marginal social cost of throughput equals the marginal social benefit. Here, since there are no externalities on the demand side, the marginal social benefit at a given level of throughput is given by the corresponding point on the demand curve. Calculating marginal social cost is complicated by the fact that per driver cost depends on the occupancy rate rather than throughput. We proceed as follows. First, we define minimized total cost as a function of throughput:

$$
\begin{gathered}
\hat{T C}(r)=\min _{x, e, Q} r[\operatorname{ETTC}(x, Q)+\operatorname{ESCD}(x, e, Q)] \\
\text { s.t. } Q P-r(L+E W(x, Q)=0
\end{gathered}
$$

where $E W(x, Q), \operatorname{ETTC}(x, Q)$, and $\operatorname{ESDC}(x, e, Q)$ are given by (1) through (4), (6), and (7). Then

$$
M \hat{S} C(r)=\frac{d \hat{T C}(r)}{d r}
$$

The above procedure calculates the direct control total cost function, assuming that the planner chooses $x$ and $e$. But it is the individual driver and not 
the planner who chooses $x$ and $e$. Thus, the total indirect control cost function should be calculated, taking into account that $x$ and $e$ are chosen by individual drivers, with the planner having only indirect control of $x$ and $e$, through the parking fee. It turns out, however, that this complication is immaterial since, when the planner chooses the curbside parking fee optimally, drivers choose the socially optimal $x$ and $e$. Thus, to simplify the analysis, I solve for the direct control social optimum, and then for the optimal parking fee that decentralizes it.

Where $X(r)$ is the total social benefit derived from $r$ trips per unit area-time (the area under the demand curve), the direct control social welfare optimization problem is

$$
\begin{gathered}
\min X(r)-r[\operatorname{ETTC}(x, Q)+\operatorname{ESDC}(x, e, Q)] \\
\text { s.t. } Q P-r(L+E W(x, Q))=0 \quad \lambda
\end{gathered}
$$

where $\lambda$ is the shadow price on this form of the steady-state condition. The first-order conditions are

$$
\begin{array}{ll}
r: & X^{\prime}-E T T C-E S D C-\lambda(L+E W)=0 \\
Q: & -r\left[E T T C_{Q}+E S D C_{Q}\right]+\lambda\left(P-r\left(E W_{Q}\right)\right)=0 \\
x: & -r\left[E T T C_{x}+E S D C_{x}\right]-\lambda r\left(E W_{x}\right)=0 \\
e: & -r\left[E S C D_{e}\right]=0
\end{array}
$$

Note four things. First, it is evident from the first-order condition with respect to $r$ that $\lambda$ is the shadow price of a curbside parking space per unit time. Second, the first-order condition with respect to $e$ is the same as the corresponding driver's first-order condition, (9). Third, from (10), the driver's first-order condition with respect to $x$ is the same as the corresponding firstorder condition for the social optimum when the curbside parking fee is set equal to the shadow price of parking. Thus, as claimed, drivers make socially 
efficient decisions with respect to both $x$ and $e$ when the curbside parking fee is set equal to the shadow price of parking. The intuition is straightforward. There is only one externality in the model, the parking externality. When this is internalized, drivers make socially efficient decisions. Fourth, the problem is decomposable. The first step entails calculating $\hat{T C}(r)$ per (18), the second step entails maximizing $X(r)-\hat{T C}(r)$, with respect to $r$.

From the first-order condition with respect to $Q$, the shadow price of a curbside parking space equals

$$
\lambda=\frac{r\left[E T T C_{Q}+E S D C_{Q}\right]}{P-r\left(E W_{Q}\right)}
$$

The interpretation of this shadow price as the parking externality cost requires some care. First, the $\lambda$ in (21) is exactly the same as the $\lambda$ in (18), since the externality is a production externality. Second, the externality operates through $Q$ and not directly through $r$. Instead a marginal increase in $r$ affects $Q$, and the marginal increase in $Q$ generates the external costs by increasing all drivers' trip cost. Third, while there are two values of $Q$ that solve the steady-state condition for a given $r$, one associated with hypercongested parking, the other with congested parking, in determining the social optimum only the congested value of $Q$ is relevant. Thus, we may express how the social cost minimizing $Q$ varies with $r$, per (18), as $Q=Q^{*}(r)$, and similarly we may write $e=e^{*}(r)$ and $x=x^{*}(r)$. Then total cost can be rewritten as

$$
\hat{T C}(r)=r\left[\operatorname{ETTC}\left(x^{*}(r), Q^{*}(r)\right)+\operatorname{ESDC}\left(x^{*}(r), e^{*}(r), Q^{*}(r)\right)\right]
$$

so that (since the derivatives with respect to $x^{*}(r)$ and $e^{*}(r)$ equal 0 via the Envelope Theorem) 


$$
\begin{aligned}
M \hat{S} C(r) & =\operatorname{ETTC}\left(x^{*}(r), Q^{*}(r)\right)+\operatorname{ESDC}\left(x^{*}(r), e^{*}(r), Q^{*}(r)\right) \\
& +\left\{\left[E T T C_{Q}\left(x^{*}(r), Q^{*}(r)\right)+E S D C_{Q}\left(x^{*}(r), e^{*}(r), Q^{*}(r)\right)\right] r Q^{*^{\prime}}(r)\right\},
\end{aligned}
$$

where, from the steady-state condition, $r Q^{*^{\prime}}(r)=(L+E W) r /\left(P-r E W_{Q}\right)$. The sum of the first two terms on the right-hand side is average trip cost so that the residual term in curly brackets is the marginal parking congestion externality cost, which is analogous to the familiar marginal congestion externality cost. Raising throughput (drivers/distance-time) by 1 unit increases the occupancy rate by $Q^{*^{\prime}}(r)$ (unitless), which raises each inframarginal driver's $\operatorname{cost}\left(\$ /\right.$ trip) by $\left[\operatorname{ETTC}_{Q}\left(x^{*}(r), Q^{*}(r)\right)+E S D C_{Q}\left(x^{*}(r), e^{*}(r), Q^{*}(r)\right)\right] Q^{*^{\prime}}(r)$ $(\$ /$ trip $)$ and hence the total cost $(\$ /$ distance-time) of inframarginal drivers by $\left[\operatorname{ETTC}_{Q}\left(x^{*}(r), Q^{*}(r)\right)+\operatorname{ESDC}_{Q}\left(x^{*}(r), e^{*}(r), Q^{*}(r)\right)\right] r Q^{*^{\prime}}(r)$. Or, put more informally, as is conventionally done, the marginal driver imposes an external cost on each of the $r$ inframargial drivers by $\left[\operatorname{ETTC}_{Q}\left(x^{*}(r), Q^{*}(r)\right)+\right.$ $\left.\operatorname{ESDC}_{Q}\left(x^{*}(r), e^{*}(r), Q^{*}(r)\right)\right] Q^{*^{\prime}}(r)$.

The social optimum is decentralized simply by setting the curbside parking fee equal to $\lambda^{*}$.

In the standard diagrammatic analysis of the traffic congestion externality, the marginal congestion externality cost equals the vertical distance between the marginal social cost of a trip and the user cost. Here, a driver's user cost depends on his choice of $x$ and $e$, which depends on the curbside parking fee. Define the user cost function when the parking fee is set equal to the value of $\lambda$, evaluated at the social optimum, $\lambda^{*}$ to be 


$$
\begin{aligned}
\hat{u c}\left(r ; \lambda^{*}\right) & =r\left[\operatorname{ETTC}\left(x\left(\hat{Q}\left(r, \lambda^{*}\right), \lambda^{*}\right), \hat{Q}\left(r, \lambda^{*}\right)\right)\right. \\
& \left.+\operatorname{ESDC}\left(x\left(\hat{Q}\left(r, \lambda^{*}\right), \lambda^{*}\right), e\left(\hat{Q}\left(r, \lambda^{*}\right), \lambda^{*}\right), \hat{Q}\left(r, \lambda^{*}\right)\right), \hat{Q}\left(r, \lambda^{*}\right)\right) \\
& +\lambda^{*}\left(L+E W\left(x\left(\hat{Q}\left(r, \lambda^{*}\right), \hat{Q}\left(r, \lambda^{*}\right)\right)\right.\right.
\end{aligned}
$$

where $\hat{Q}\left(r, \lambda^{*}\right)$ corresponds to the congested solution to the steady-state condition when the curbside parking fee is set equal to $\lambda^{*}$.

Figure 3 presents the social optimum diagrammatically in a form familiar from the analysis of congestion pricing. The social optimum occurs at the intersection of the demand curve and the marginal social cost curve, msc. The curve $u c$ is the user cost curve when the curbside parking fee is set at the optimal level, per the above definition. When the curbside parking fee is set at the optimal level, the supply curve is the user cost curve shifted up by $\lambda^{*}(L+$ $E W\left(x\left(\hat{Q}\left(r, \lambda^{*}\right), \hat{Q}\left(r, \lambda^{*}\right)\right)\right.$. And the equilibrium when the curbside parking fee is set at the optimal level coincides with the optimum. 
Figure 3: Decentralization of the social optimum.

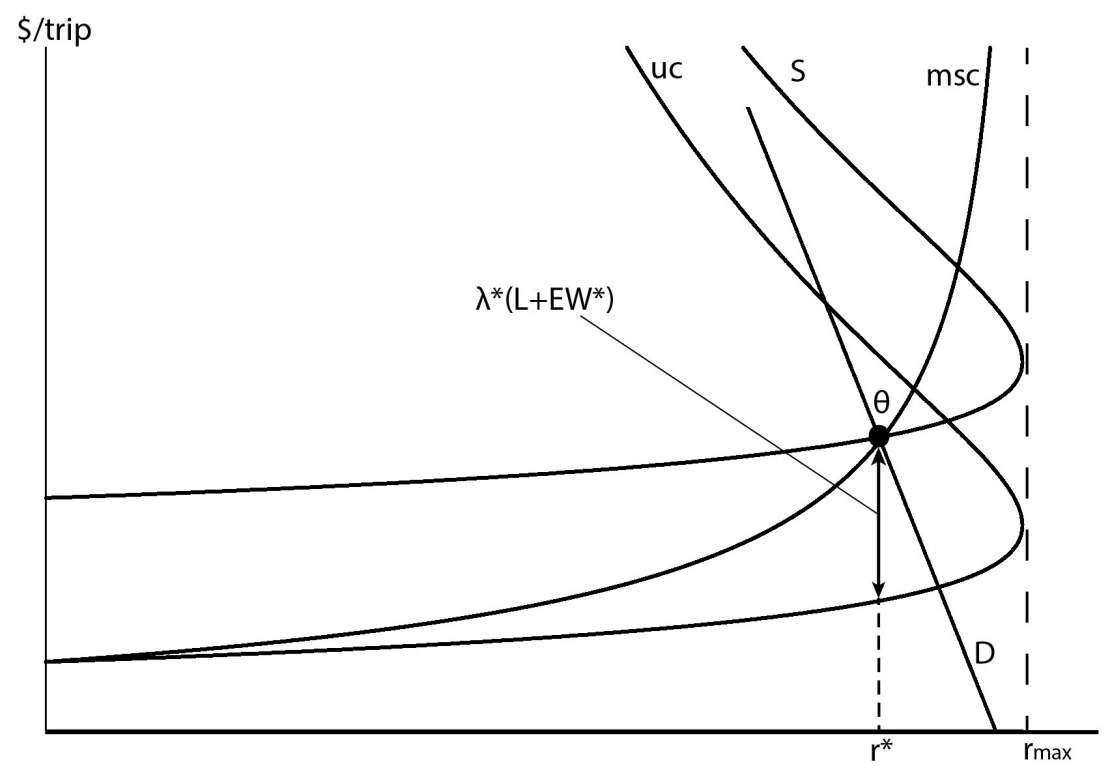

Note: The diagram is qualitative. It is not drawn by graphing functions of the model for specific parameter values.

The comparative static derivatives of the social optimum with respect to $\eta$, which we term demand intensity and which corresponds to an outward shift in demand are

$$
\begin{gathered}
\frac{d r}{d \eta}>0, \frac{d Q}{d \eta}>0, \frac{d x}{d \eta} ?, \frac{d e}{d \eta} ?, \frac{d M(x, e)}{d \eta}>0 \\
\frac{d \lambda}{d \eta}>0, \frac{d F}{d \eta}>0
\end{gathered}
$$

Note that the increase in $\eta$ affects the driver's choice of $x$ and $e$ through an increase in both $Q$ and $f$.

One result is of paramount importance - the socially optimal target curbside parking occupancy rate is increasing in demand intensity ${ }^{9}$. Thus, the search for a

\footnotetext{
${ }^{9}$ It is tempting to generalize from this result to real-world situations, arguing for example that the occupancy rate should be higher during peak than off-peak hours, or at more rather than less congested locations. I caution the reader against doing so. First, the real world in more complex than the model here, including intra-day traffic dynamics, heterogeneity of drivers and street space, and two-dimensional rather than one-dimensional search. Second,
} 
universal socially optimal target curbside parking occupancy rate is a will o' the wisp. The intuition is straightforward. The shift upwards in the marginal social benefit curve implies an increase in the optimal throughput, which requires an increase in the occupancy rate.

Most other comparative static derivatives are ambiguous in sign. For one thing, from (20) most contain terms in $E T T C_{Q Q}, E S D C_{Q Q}$, and/or $E W_{Q Q}$, each of which depends on $G_{Q Q}$, about which no assumption has been made ${ }^{10}$.

\section{Directions to Future Research}

In discussing directions for research, I have in mind what would need to be done to extend the model for use in practical applications, such as SFpark.

\section{- Incorporating traffic congestion}

The interaction between curbside parking and traffic congestion is practically important, and should be considered in any model that aims to derive a realistic optimal target curbside parking occupancy rate. In a series of related papers, Arnott and Inci (2006, 2010), Arnott and Rowse (2009, forthcoming), and Arnott et al. (2013), Arnott, Inci, and Rowse have developed a sequence of related models that treat the interaction between curbside parking, garage parking, and traffic congestion in a isotropic, two-dimensional area in steady state $^{11}$. The model of this paper could be augmented to include the interaction

the result relates to a change in a single exogenous variable, $\eta$, whereas in the real world, exogenous variables need not change one at a time; for example, more congested locations may have a systematically lower or higher curbside parking density, and peak-period drivers may have a higher value of time than off-peak drivers. Third, there are scale effects. One might think that the optimal occupancy rate depends on the ratio of demand intensity to curbside parking capacity. But this is incorrect since a doubling of curbside parking capacity implies a doubling of the number of parking spaces visited within a given distance.

Nevertheless, since negative results generalize form more specific to more general settings, one my safely conclude that, in real-world situations, the optimal target curbside occupancy rate is not constant over time and space.

${ }^{10}$ Rather obviously, a doubling of all exogenous monetary values has no effect on the optimum $r, Q, x, e$, and $M$, but causes a doubling of $F$.

${ }^{11}$ The papers provide an unsophisticated treatment of curbside parking search. Either parking is unsaturated everywhere, in which case curbside parking search costs are zero, or 
between curbside parking and traffic congestion in the same way as was done in Arnott and Inci (2006). Curbside parking interacts with traffic congestion in two important ways. Having parking curbside reduces the road space available for traffic flow, and cars searching for parking contribute to traffic congestion.

With traffic congestion added, the optimal amount of curbside to allocate to parking (optimal curbside parking capacity) can be determined both when traffic congestion is efficiently priced and when it is not, as was done in Arnott et al. (2013) with their less sophisticated treatment of traffic congestion. For each level of demand, social surplus is solved for as a function of curbside parking capacity, with account being taken that drivers decide on trip frequency, as well as $x$ and $e$, taking the occupancy rate and curbside parking fee as fixed, so as to maximize their private surplus.

\section{- Garage parking}

Arnott and Rowse (2009) added private garage parking to the Arnott-Inci model of downtown parking and traffic congestion. That paper ignores that costs of searching for parking inside parking garages, and provides two treatments of private garage location and costs. In the simpler treatment, garage parking is provided continuously over space at constant cost and priced at this cost (Bertrand competition). In the more sophisticated model, garages are discretely spaced due to economies of scale in garage construction, and consequently have market power. Garage parking could be introduced into the model of this paper in either of these two ways. Since SFpark is adjusting parking prices so as to achieve a target occupancy rate not only curbside but also in public parking garages, the model would be more useful if it were extended to treat search for parking inside parking garages, as well as public garages.

parking is saturated everywhere, in which case expected curbside parking search time equals the curbside parking turnover rate per unit area divided by the stock of cars cruising for parking per unit area. 


\section{- Rush-hour dynamics}

One earlier paper, Arnott et al. (1991), and several recent papers (Qian et al. (2012), Zhang et al. (2008) and Zhang et al. (2011)) have been written that extend Vickrey's bottleneck model of rush-hour traffic congestion to include parking. The papers provide different simplified treatments of parking and cruising for parking. The model of this paper, with its more sophisticated treatment of parking, could be extended relatively straightforwardly to treat rush-hour traffic and parking dynamics via the bottleneck model. Doing so would permit investigation of whether the target curbside parking occupancy rate should differ according to the stage of the rush hour.

\section{- Two-dimensional space}

This paper had two main goals. The first was to develop a structural model of curbside parking that has the potential of being extended to the point where it can be applied in practical policy contexts. The second was to make the general point that there is no universal optimal target curbside parking occupancy rate. The paper has, I think, achieved these modest goals. But the model is still far from practical application. One of its most obvious deficiencies is that it almost trivializes the curbside parking search problem by treating it as one-dimensional. But practically curbside parking search is two-dimensional, and two-dimensional parking search is much more difficult to treat than one-dimensional parking search. A first step in analyzing two-dimensional parking search is to model it in an isotropic space (an infinite plain or the surface of a sphere). A driver's optimal parking search strategy (e.g., drive to the destination block, and cruise around the block until a parking space opens up) depends on other drivers' search strategies, which suggests that there may be multiple equilibria. 


\section{- Anisotropic space}

The monocentric city is perhaps the simplest interesting anisotropic space. Arnott et al. (1991) and Anderson and de Palma (2004) have analyzed curbside parking in the monocentric city model. Drivers park from the CBD either inwards or outwards depending on the parking fee structure. Parking inwards is more efficient since it concentrates the distribution of arrivals, conditional on the distribution of departures. While analysis of curbside parking in the monocentric city model generates important general insight, for policy purposes what is of interest is the actual street network, which requires downtown traffic microsimulation models to deal with. Thus, an important topic on the research agenda is to strengthen these models' parking modules, for instance by accommodating heterogeneity in search strategy.

\section{- Heterogeneity}

Heterogeneity is potentially important in any nonlinear model. It remains to be seen how important driver heterogeneity is in determining the optimal target curbside parking occupancy rate.

\section{- Estimating the $g(z ; Q)$ function}

SFpark is collecting comprehensive data on the occupancy histories of public parking garages and individual parking meters. These data are insufficient to measure social surplus. To do this, it is necessary to relate aggregate travel costs to the observables, such as the curbside occupancy rate and the distribution of curbside parking times. The model indicates that the form of $g(z ; Q)$ is central to this relationship. All previous theoretical work has assumed this function to be negative exponential (where $z$ is treated as continuous) or binomial (where $z$ is treated as discrete). But Levy et al. (2012) persuasively demonstrates that this assumption may lead to a severe downward bias in the estimation of curbside parking search time as a function of the occupancy rate. The form of 
this function depends on the curbside parking search strategy. Determining its form analytically under alternative parking search strategies appears to be very difficult. A more promising avenue is to estimate it from microsimulation, for first isotropic networks, then different ideal types of anisotropic networks, and then real networks.

- Curbside capacity, parking time limits, and the nonlinear pricing of curbside parking

Recent policy discussion has focused on cashing out free and heavily subsidized curbside parking by raising meter rates until curbside occupancy rate targets are met, with little attention being paid to other aspects of curbside parking policy, such as curbside parking capacity, curbside parking time limits, and the nonlinear pricing of curbside parking. In an extended calibrated numerical example, Arnott et al. (2013) illustrate that, in heavily congested downtown areas where garage parking is available, it may be optimal to eliminate curbside parking altogether, at least on the most congested streets, especially when curbside parking is substantially underpriced. When drivers differ in visit length and the value of time, when curbside parking is underpriced, and when garage parking is available, Arnott and Rowse (forthcoming) show that curbside parking time limits both decrease cruising for parking and increase the parking turnover rate, both of which make shopping downtown more attractive. Curbside parking time limits and a curbside parking meter rate generate a form of non-linear pricing of curbside parking. Today's hi-tech parking meters permit the implementation of arbitrary non-linear curbside parking pricing.

\section{Conculsion}

Policy makers are coming to recognize the importance of parking policy in the management of downtown auto congestion. One aspect of parking policy that has recently received considerable attention is efficient curbside parking pricing. 
The general rule is that the market-clearing price is the efficient price. This rule applies to curbside parking, but because of the stochasticity associated with curbside parking entry and exit, its implementation would require responsive pricing, in which the parking fee on a particular block at a particular time of day would depend on the particular realization of the stochastic process. Such pricing would be informationally demanding, hard to implement, and annoying to drivers. A more practical policy is to set curbside parking fees ex ante. The efficient ex ante fee would balance the efficiency cost of having the fee below its market-clearing level when realized demand is high (cruising for parking costs) and the efficiency cost of having the fee above its market-clearing level when demand is low (unutilized curbside parking capacity).

How should the efficient ex ante curbside parking fee on a particular block and for a particular period of the day be determined in practice? Donald Shoup has advocated setting block- and time-period specific fees so that a common average curbside parking occupancy rate is achieved. The cities of San Francisco and Los Angeles are implementing Shoup's proposal on an experimental basis (SFpark and LA Express Park, respectively).

This paper developed a simple, structural model of curbside parking to investigate the theoretical basis for an optimal target curbside parking occupancy rate rule. Parking takes place on the outside of a circle, and only the (stochastic) steady state is analyzed. The analysis contained three elements. The first solved for drivers' optimal strategy in cruising for parking, taking as given the probability function for the number of curbside parking spaces searched before a vacant space is found, which depends on the curbside occupancy rate, as well as the curbside parking fee. The second solved for steady-state equilibrium in which, in expectation, occupied parking spaces per unit length (the occupancy rate times the density of parking spaces per unit length) equals throughput 
per unit length times parking duration (visit duration plus time walking between the parking space and the destination). And the third solved for the social optimum. Analogously to Walters' well-known diagrammatic analysis of steady-state traffic congestion (1961), at the optimum the marginal social cost of throughput equals the marginal social benefit, and the optimum can be decentralized by imposing a parking fee such that a driver faces the marginal social cost of a trip. The corresponding optimal target curbside parking occupancy rate is higher, the higher is realized demand intensity.

The paper provides the conceptual basis for not only determining the optimal target curbside parking occupancy rate, but also for undertaking welfare analysis of policies related to curbside parking. Much remains to be done, however, in extending the model in the direction of realism, before it can usefully be implemented in specific policy contexts, as was discussed in section 5 .

SFpark is gradually adjusting parking fees by lock until target curbside occupancy rates are achieved. Comprehensive data are being collected on the occupancy experience of every parking meter and every public parking garage in the programs. But to undertake welfare analysis, it is necessary to relate occupancy rates to driver costs, including cruising-for-parking time costs, walking costs, and schedule delay costs. One approach is to collect the data needed to estimate these relationships directly. An alternative approach is to apply a structural model, such as an extended version of this paper's model. A crucial element of both approaches is to estimate cruising-for-parking search strategies.

\section{Acknowledgments}

I would like to thank Mehdi Naji for very capable research assistance and participants at the OPLOG seminar in the Sauder School of Business, University of British Columbia, for helpful comments. 


\section{Appendix A. $E S D C_{x}$}

\section{(NOT INTENDED FOR PUBLICATION)}

To avoid the need to flip backwards, we repeat (6) and (7):

$$
\begin{aligned}
\operatorname{ESDC}(x, e ; Q)= & \beta \int_{0}^{x}\left[e-\frac{\delta}{v}+z\left(\frac{1}{w}-\frac{1}{s}\right)-x\left(\frac{1}{w}-\frac{1}{v}\right)\right] g(z ; Q) d z \\
& +\beta \int_{x}^{M(x, e)}\left[e-\frac{\delta}{v}-z\left(\frac{1}{w}+\frac{1}{s}\right)+x\left(\frac{1}{w}+\frac{1}{v}\right)\right] g(z ; Q) d z \\
& +\gamma \int_{M(x, e)}^{\infty}\left[-e+\frac{\delta}{v}+z\left(\frac{1}{w}+\frac{1}{s}\right)-x\left(\frac{1}{w}+\frac{1}{v}\right)\right] g(z ; Q) d z
\end{aligned}
$$

where

$$
M(x, e)=\left[e-\frac{\delta}{v}+x\left(\frac{1}{v}+\frac{1}{w}\right)\right]\left[\frac{1}{s}+\frac{1}{w}\right]^{-1}
$$

Note that

$$
M_{x}(x, e)=\left(\frac{1}{v}+\frac{1}{w}\right)\left[\frac{1}{s}+\frac{1}{w}\right]^{-1}>0 .
$$

Then 


$$
\begin{aligned}
& E S D C_{x}(x, e ; Q)=\beta\left[-\left(\frac{1}{w}-\frac{1}{v}\right) G(x ; Q)+\left(\frac{1}{w}+\frac{1}{v}\right)(G(M(x, e) ; Q)-G(x ; Q)]\right. \\
& -\gamma\left[\left(\frac{1}{w}+\frac{1}{v}\right)(1-G(M(x, e) ; Q))\right]+\beta\left[-\left(\frac{1}{s}-\frac{1}{v}\right) x g(x ; Q)+\left(\frac{1}{s}-\frac{1}{v}\right) x g(x ; Q)\right] \\
& +\beta\left[e-\frac{\delta}{v}-M(x, e)\left(\frac{1}{w}+\frac{1}{s}\right)+x\left(\frac{1}{w}+\frac{1}{v}\right)\right] g(M(x, e) ; Q) M_{x}(x, e) \\
& -\gamma\left[-e+\frac{\delta}{v}+M(x, e)\left(\frac{1}{w}+\frac{1}{s}\right)-x\left(\frac{1}{w}+\frac{1}{v}\right)\right] g(M(x, e) ; Q) M_{x}(x, e) \\
& =\beta\left[-\left(\frac{1}{w}-\frac{1}{v}\right) G(x ; Q)+\left(\frac{1}{w}+\frac{1}{v}\right)(G(M(x, e) ; Q)-G(x ; Q)]\right. \\
& -\gamma\left[\left(\frac{1}{w}+\frac{1}{v}\right)(1-G(M(x, e) ; Q))\right] \\
& +\beta\left[e-\frac{\delta}{v}-M(x, e)\left(\frac{1}{w}+\frac{1}{s}\right)+x\left(\frac{1}{w}+\frac{1}{v}\right)\right] g(M(x, e) ; Q) M_{x}(x, e) \\
& -\gamma\left[-e+\frac{\delta}{v}+M(x, e)\left(\frac{1}{w}+\frac{1}{s}\right)-x\left(\frac{1}{w}+\frac{1}{v}\right)\right] g(M(x, e) ; Q) M_{x}(x, e) \\
& =\beta\left[-\left(\frac{1}{w}-\frac{1}{v}\right) G(x ; Q)+\left(\frac{1}{w}+\frac{1}{v}\right)(G(M(x, e) ; Q)-G(x ; Q)]\right. \\
& -\gamma\left[\left(\frac{1}{w}+\frac{1}{v}\right)(1-G(M(x, e) ; Q))\right] \\
& +\beta e-\frac{\delta}{v}-\left[e-\frac{\delta}{v}+x\left(\frac{1}{v}+\frac{1}{w}\right)\right]+x\left(\frac{1}{w}+\frac{1}{v}\right) g(M(x, e) ; Q) M_{x}(x, e) \\
& -\gamma-e+\frac{\delta}{v}+\left[e-\frac{\delta}{v}+x\left(\frac{1}{v}+\frac{1}{w}\right)\right]-x\left(\frac{1}{w}+\frac{1}{v}\right) g(M(x, e) ; Q) M_{x}(x, e) \\
& =\beta\left[-\left(\frac{1}{w}-\frac{1}{v}\right) G(x ; Q)+\left(\frac{1}{w}+\frac{1}{v}\right)(G(M(x, e) ; Q)-G(x ; Q)]\right. \\
& -\gamma\left[\left(\frac{1}{w}+\frac{1}{v}\right)(1-G(M(x, e) ; Q))\right] \\
& =-2 \frac{\beta G(x ; Q)}{w}+\left(\frac{1}{w}+\frac{1}{v}\right)(\beta G(M(x, e))-\gamma(1-G(M(x, e)))
\end{aligned}
$$

\section{Appendix B. The Primitive Necessary and Sufficient Condition for A-1}

(NOT INTENDED FOR PUBLICATION)

Recall that $a(x, e, z)$ is defined to be the arrival time of a driver who departs a period of time $e$ before her appointment, starts cruising for parking a distance $x$ before her destination, and cruises for parking a distance $z$ before finding her 
first vacant parking space, and is given by

$$
a(x, e, z)=-e+\frac{\delta-x}{v}+\frac{z}{s}+\frac{|z-x|}{w}
$$

Recall too

A-1: $a(x, e, 0)=-e+\frac{\delta-x}{v}+\frac{x}{w}<0$

This assumption states that a driver who finds a parking space as soon as she starts cruising for parking arrives early. This appendix derives the necessary and sufficient condition on parameter values and functional forms such that A-1 holds. Where $A=1 / w-1 / v, \mathbf{A - 1}$ can be rewritten as

$$
\frac{\delta}{v}<e-x A .
$$

We shall derive $e$ and $x$ as functions of exogenous parameters using the firstorder conditions for them, and then substitute these functions in (B.1). The first-order condition with respect e is given by (9) and that with respect to $x$ is given by (13).

Recall that $M(x, e)$ is the distance cruising for parking consistent with ontime arrival, i.e. $a(x, e, M(x, e))=0$. Under $\mathbf{A - 1}$, since the driver who parks at his destination arrives early, the driver who arrives exactly on time parks beyond her destination, from (5)

$$
M(x, e)=\left(e-\frac{\delta}{v}+x B\right) \frac{1}{C}
$$

where $B=(1 / v+1 / w)$ and $C=(1 / s+1 / w)$.

Substituting (7) into (9), the first-order condition with respect to $e$ is

$$
\left(e-\frac{\delta}{v}+x B\right) \frac{1}{C}=G^{-1}\left\{\frac{\gamma}{\beta+\gamma}\right\}
$$


From (14), which is obtained from substituting (B.2) into (13), the first-order condition with respect to $x$ is

$$
x=G^{-1}\left\{\frac{\alpha+f+\frac{\alpha w}{v}}{2(\alpha+f)-\beta}\right\}
$$

Substituting (B.3) into (B.2) yields

$$
\begin{aligned}
e & =\frac{\delta}{v}-x B+C G^{-1}\left\{\frac{\gamma}{\beta+\gamma}\right\} \\
& =\frac{\delta}{v}-B G^{-1}\left\{\frac{\delta+f+\frac{\alpha w}{v}}{2(\alpha+f)-\beta}\right\}+C G^{-1}\left\{\frac{\gamma}{\beta+\gamma}\right\}
\end{aligned}
$$

Substituting (B.3) and (B.4) into (B.1) yields

$$
-B G^{-1}\left\{\frac{\alpha+f+\frac{\alpha w}{v}}{2(\alpha+f)-\beta}\right\}+C G^{-1}\left\{\frac{\gamma}{\beta+\gamma}\right\}-A G^{-1}\left\{\frac{\alpha+f+\frac{\alpha w}{v}}{2(\alpha+f)-\beta}\right\}>0
$$

Since $B+A=2 / w$, this reduces to

$$
-\frac{2}{w} G^{-1}\left\{\frac{\alpha+f+\frac{\alpha w}{v}}{2(\alpha+f)-\beta}\right\}+C G^{-1}\left\{\frac{\gamma}{\beta+\gamma}\right\}>0
$$

or

$$
\frac{1}{2}\left(1+\frac{w}{s}\right)>\frac{G^{-1}\left\{\frac{\alpha+f+\frac{\alpha w}{v}}{2(\alpha+f)-\beta}\right\}}{G^{-1}\left\{\frac{\gamma}{\beta+\gamma}\right\}} .
$$

\section{Appendix C. Comparative Statics of the Driver's Optimization Prob- lem}

\section{(NOT INTENDED FOR PUBLICATION)}

From (9), the first-order condition with respect to $e$ is

$$
(\gamma+\beta) G(M(x, e) ; Q)-\gamma=0
$$


where, recall,

$$
M(x, e)=\left[e-\frac{\delta}{v}+x\left(\frac{1}{v}+\frac{1}{w}\right)\right]\left[\frac{1}{s}+\frac{1}{w}\right]^{-1}
$$

From (14), the first-order condition with respect to $x$, after the first-order condition with respect to $e$ has been substituted in, is

$$
[2(\alpha+f)-\beta] G(x ; Q)-\left[(\alpha+f)+\frac{\alpha w}{v}\right]=0
$$

We denote the $x$ and $e$ that solve (C.1) and (C.2) by $x(f, Q)$ and $e(f, Q)$.We are interested in the effects of changes in $f$ and $Q$ on $e$ and $x$. Totally differentiating (C.1) and (C.2) yields

$$
\begin{aligned}
& (\gamma+\beta) g(M(x, e) ; Q) M_{e}(x, e) d e \\
& +(\gamma+\beta) g(M(x, e) ; Q) M_{x}(x, e) d x \\
& =-(\gamma+\beta) G_{Q}(M(x, e) ; Q) d Q+0 d f
\end{aligned}
$$

or

$$
\begin{aligned}
& (\gamma+\beta) g(M(x, e) ; Q)\left[\frac{1}{s}+\frac{1}{w}\right]^{-1} d e \\
& +(\gamma+\beta) g(M(x, e) ; Q)\left(\frac{1}{v}+\frac{1}{w}\right)\left[\frac{1}{s}+\frac{1}{w}\right]^{-1} d x \\
& =-(\gamma+\beta) G_{Q}(M(x, e) ; Q) d Q+0 d f
\end{aligned}
$$

$$
\begin{aligned}
& 0 d e+[2(\alpha+f)-\beta] g(x ; Q) d x \\
& =-[2(\alpha+f)-\beta] G_{Q}(x ; Q) d Q+(1-2 G(x ; Q)) d f
\end{aligned}
$$


Since $x$ and $e$ are the endogenous variables, and $Q$ and $f$ the exogenous variables, we obtain from (C.3) and (C.4) that

$$
\begin{aligned}
x_{Q} & =-\frac{G_{Q}(x ; Q)}{g(x ; Q)}>0 \\
x_{f} & =\frac{1-2 G(x ; Q)}{(2(\alpha+f)-\beta) g(x ; Q)]} \\
& =-\frac{\beta+\frac{2 \alpha w}{v}}{[2(\alpha+f)-\beta]^{2}}<0, \operatorname{using}(14) \\
e_{f} & =-\left(\frac{1}{v}+\frac{1}{w}\right) x_{f}>0
\end{aligned}
$$

Also, from (C.3),

$$
\begin{aligned}
& g(M(x, e) ; Q)\left[\frac{1}{s}+\frac{1}{w}\right]^{-1} e_{Q} \\
& \left.+g(M(x, e) ; Q)\left(\frac{1}{v}+\frac{1}{w}\right)\right]\left[\frac{1}{s}+\frac{1}{w}\right]^{-1} x_{Q} \\
& =-G_{Q}(M(x, e) ; Q)
\end{aligned}
$$

so that, from (C.5)

$$
\begin{aligned}
e_{Q} & =-\left(\frac{1}{v}+\frac{1}{w}\right) x_{Q}-\frac{G_{Q}(M(x, e) ; Q)\left[\frac{1}{s}+\frac{1}{w}\right]}{g(M(x, e) ; Q)} \\
& =\left(\frac{1}{v}+\frac{1}{w}\right) \frac{G_{Q}(x ; Q)}{g(x ; Q)}-\left(\frac{1}{s}+\frac{1}{w}\right) \frac{G_{Q}(M(x, e) ; Q)}{g(M(x, e) ; Q)}
\end{aligned}
$$

which is of ambiguous sign.

\section{Appendix D. Comparative Static Properties of Equilibrium}

\section{(NOT INTENDED FOR PUBLICATION)}

We write the equations characterizing equilibrium as 


$$
\begin{aligned}
& \operatorname{ETTC}(x(Q, f), Q)+E S D C(x(Q, f), e(Q, f), Q) \\
& +f(L+E W(x(Q, f), Q))-F=0 \\
& D(F ; \eta)-r=0 \\
& (L+E W(x(Q, f), Q) r-Q P=0
\end{aligned}
$$

We do this since we are interested in the effects of exogenous variables not only on $F$ and $Q$, but also on $r$, since social benefit depends on the level of throughput, and since this formulation facilitates the presentation of results in the familiar terms of supply and demand ( $r-F$ space). Note that $\eta$ is a demand shifter, with an increase in $\eta$ corresponding to an increase in demand.

We are interested in the effect of an increase in the meter rate and also of demand on the equilibrium. This system looks somewhat complicated, but note that the individual chooses $x$ and $e$ to minimize $F$, so that the first-order derivatives with respect to $x$ and $e$ in the first equation can be ignored. Total differentiation of the system of equations yields

$$
\left[\begin{array}{ccc}
A & 0 & -1 \\
0 & -1 & D^{\prime} \\
B & L+E W & 0
\end{array}\right]\left[\begin{array}{c}
d Q \\
d r \\
d F
\end{array}\right]=\left[\begin{array}{c}
-(L+E W) \\
0 \\
-E W_{x} x_{f}
\end{array}\right] d f+\left[\begin{array}{c}
0 \\
-D_{\eta} \\
0
\end{array}\right] d \eta
$$

where

$$
A=E T T C_{Q}+E S D C_{Q}+f E W_{Q} \text { and } B=\left(E W_{x} x_{Q}+E W_{Q}\right) r-P
$$


Now,

$$
\begin{aligned}
\Delta= & -\left[\left(E W_{x} x_{Q}+E W_{Q}\right) r-P\right] \\
& -D^{\prime}(L+E W)\left[E T T C_{Q}+E S D C_{Q}+f E W_{Q}\right]
\end{aligned}
$$

Under a reasonable adjustment process ${ }^{12}$, equilibria can be shown to be stable when $\Delta$ is positive and unstable when it is negative.

We obtain

$$
\begin{aligned}
\frac{d Q}{d \eta} & =\frac{D_{\eta}(L+E W)}{\Delta} \\
\frac{d r}{d \eta} & =-\frac{\left[\left(E W_{x} x_{Q}+E W_{Q}\right) r-P\right] D_{\eta}}{\Delta} \\
\frac{d F}{d \eta} & =\frac{D_{\eta}(L+E W)\left[E T T C_{Q}+E S D C_{Q}+f E W_{Q}\right]}{\Delta}
\end{aligned}
$$

These results are consistent with Figure 2. At a stable equilibrium, an increase in demand causes an increase in the steady-state occupancy rate, and an increase in the full price of a trip. Intuitively, an increase in demand should increase throughput iff the elasticity of the average time parked with respect to $Q$ is less than 1.0. This is confirmed by rewriting the equation for $d r / d \eta$ in (D.3) as

\footnotetext{
${ }^{12} \mathrm{~A}$ natural adjustment process has the drivers entering the city at a rate based on the expected full price, which can be based on myopic expectations or perfect foresight, and exiting according to the model description. Arnott and Inci (2010) formally examine the stability of an analogous model, with the modification that visit lengths have a negative exponential distribution.
} 


$$
\begin{aligned}
\frac{d r}{d \eta} & =\frac{-\left[\left(E W_{x} x_{Q}+E W_{Q}\right) r-P\right] D_{\eta}}{\Delta} \\
& =\left[\frac{-r D_{\eta}}{\Delta}\right]\left[\left(E W_{x} x_{Q}+E W_{Q}\right)-\frac{(L+E W)}{Q}\right] \\
& =\left[\frac{-r D_{\eta}(L+E W)}{\Delta Q}\right]\left[\frac{\left(E W_{x} x_{Q}+E W_{Q}\right) Q}{(L+E W)}-1\right]
\end{aligned}
$$

The term in the first square brackets on the RHS is negative, and that in the second square brackets is negative iff the elasticity of average parking time with respect to $Q$ is less than one, which is the case at a congested equilibrium.

$$
\begin{gathered}
\frac{d Q}{d f}=\frac{\left[D^{\prime}(L+E W)^{2}+E W_{x} x_{f}\right]}{\Delta}<0 \\
\frac{d r}{d f}=\frac{\left[-(L+E W) D^{\prime}\left[\left(E W_{x} x_{Q}+E W_{Q}\right) r-P\right]+E W_{x} x_{f}\left[E T T C_{Q}+E S D C_{Q}+f E W_{Q}\right] D^{\prime}\right]}{\Delta} \\
\frac{d F}{d f}=\frac{-\left[\left(E W_{x} x_{Q}+E W_{Q}\right) r-P\right](L+E W)+E W_{x} x_{f}\left[E T T C_{Q}+E S D C_{Q}+f E W_{Q}\right]}{\Delta}
\end{gathered}
$$

The comparative static derivatives with respect to $f$ are more complicated since the change in the parking fee affects the endogenous variables not only directly but also through $Q, x$, and $e$. Totally differentiating (15), the full price equation, gives

$$
\frac{d F}{d f}=\left[L+E W(x(Q, f), Q]+\left[E T T C_{Q}+E S D C_{Q}+f E W_{Q}\right] \frac{d Q}{d f}\right.
$$

via the Envelope Theorem. Thus, in terms of Figure 2, a change of $d f$ in the parking fee shifts up (15) by $d f$ times the parking duration. Totally differentiating the steady-state, (17), equation yields 


$$
E W_{x} x_{f}+\left[L+E W(x(Q, f), Q] \frac{d r}{d f}+\left[E W_{x} x_{Q}+E W_{Q}-P\right] \frac{d Q}{d f}=0\right.
$$

It was established in Appendix $\mathrm{C}$ that $x_{f}<0$, and elsewhere that $E W_{x}>$ 0 . Thus, holding the occupancy rate constant, an increase in the parking fee causes throughput to increase because expected walking time and hence parking duration decrease. An increase in the parking fee by $d f$ causes throughput to increase by $-E W_{x} x_{f} /[L+E W(x(Q, f), Q]$, and, in terms of Figure 2, (17) to shift to the right.

We know too that $E T T C_{Q}+E S D C_{Q}+f E W_{Q}>0, E W_{x} x_{Q}+E W_{Q}-P>$ 0 under congestion, and $x_{Q}>0$. Thus, at a stable, congested equilibrium, $d Q / d f<0$. The sign of $d r / d f$ is opposite to the sign of $d F / d f$ since equilibrium lies on the negatively-sloped demand curve, which is unchanged by the parking fee increase. But without further assumptions, their signs are indeterminate. It is not their indeterminacy that is surprising, but the unambiguous sign of $d Q / d f$.

If $d Q / d f$ is ambiguous, by continuity it would be possible for $d Q / d f$ to equal zero. Suppose this is the case. From the stationary state condition, the increase in the curbside parking fee would then cause visit duration to fall, requiring a rise in throughput, and hence a decrease in the full price. But from the full price equation and the Envelope Theorem applied to $x(Q, f)$ and $e(Q, f)$, a rise in the curbside parking fee with no change in the occupancy rate leads to an increase in the full price of a trip, a contradiction.

\section{References}

Anderson, S.P., de Palma, A., 2004. The economics of pricing parking. Journal of Urban Economics 55, 1-20. 
Arnott, R., Inci, E., 2006. An integrated model of downtown parking and traffic congestion. Journal of Urban Economics 60, 418-442.

Arnott, R., Inci, E., 2010. The stability of downtown parking and traffic congestion. Journal of Urban Economics 68, 260-276.

Arnott, R., de Palma, A., Lindsey, R., 1991. A temporal and spatial equilibrium analysis of commuter parking. Journal of Public Economics 45, 301-335.

Arnott, R., Rowse, J., 1999. Modeling parking. Journal of Urban Economics $45,97-124$.

Arnott, R., Rowse, J., 2009. Downtown parking in auto city. Regional Science and Urban Economics 39, 1-14.

Arnott, R., Rowse, J., forthcoming. Curbside parking time limits. Transportation Research A .

Arnott, R.J., Inci, E., Rowse, J., 2013. Downtown curbside parking capacity. Technical Report. CESifo Working Paper: Public Finance 4085.

Levy, N., Martens, K., Benenson, I., 2012. Exploring cruising using agent-based and analytical models of parking. Transportmetrica 0, 1-25. doi:10.1080/ 18128602.2012 .664575 .

Qian, Z.S., Xiao, F.E., Zhang, H., 2012. Managing morning commute traffic with parking. Transportation Research Part B 46, 894-916.

Shoup, D.C., 1999. The trouble with minimum parking requirements. Transportation Research Part A 33, 549-574.

Vickrey, W., 1954. The economizing of curb parking space. Traffic Engineering, November 29, 62-67. 
Walters, A.A., 1961. The theory and measurement of private and social cost of highway congestion. Econometrica 29, 676-699.

Zhang, X., Huang, H.J., Zhang, H., 2008. Integrated daily commuting patterns and optimal road tolls and parking fees in a linear city. Transportation Research Part B 42, 38-56.

Zhang, X., Yang, H., Huang, H.J., 2011. Improving travel efficiency by parking permits distribution and trading. Transportation Research Part B 45, 10181034 . 